\title{
Analyse de la polysémie verbale : apports à la didactique du français L2 \\ Leslie Redmond ${ }^{1, *}$ et Louisette Emirkanian ${ }^{2}$
}

${ }^{1}$ Faculty of Education, Memorial University of Newfoundland

${ }^{2}$ Département de linguistique, Université du Québec à Montréal

\begin{abstract}
Résumé. Cette contribution se situe à l'interface de la linguistique et de la didactique des langues secondes; elle a pour objectif de démontrer les apports d'une analyse de la polysémie verbale à la didactique du français langue seconde. Notre premier objectif est de décrire la polysémie du verbe prendre au moyen d'une analyse sémantique lexicale dans une approche cognitive. Notre second objectif est d'évaluer l'incidence de la polysémie du verbe prendre sur les connaissances qu'ont les apprenants de ce verbe et d'isoler les différentes acceptions de prendre mises au jour par notre analyse sémantique, qui s'avèrent problématiques pour les apprenants du français L2. Pour atteindre le second objectif de notre travail, nous avons mené une étude empirique auprès de 191 apprenants du français langue seconde. Les résultats montrent non seulement que l'analyse sémantique que nous avons proposée permet de prédire la connaissance des différentes acceptions du verbe par les apprenants du français L2, mais aussi que les apprenants anglophones et allophones ont un comportement différent par rapport aux types d'acceptions du verbe prendre, comportement que nous avons pu expliquer par l'influence translangagière chez les participants anglophones. Nous discutons des résultats au regard de ceux des études antérieures, en mettant l'accent sur les variables linguistiques réputées prédire l'acquisition des différents sens d'un mot polysémique.
\end{abstract}

\begin{abstract}
An Analysis of Verbal Polysemy: Contributions to Second Language Pedagogy This paper is at the interface of linguistics and second language pedagogy; it aims to demonstrate the contributions of an analysis of verbal polysemy to the teaching of French as a second language. On the one hand, the first objective is to describe the polysemy of prendre through a lexical semantic analysis within a cognitive framework. On the other hand, the second objective is to evaluate the impact of this polysemy on learners' knowledge of the verb's different senses in order to isolate those which are the most problematic for French L2 learners. In order to meet the second objective, we conducted an experiment on 191 learners of French as a second language. The results show not only that the semantic analysis proposed can predict participants' knowledge of the different senses of the verb, but also that participants whose first language is English behave differently from those whose first language is another language, confirming the presence of cross-linguistic influence for the English-speaking participants. We discuss results in light of previous findings, focusing on the linguistic variables shown to predict the acquisition of different meanings of a polysemous word.
\end{abstract}

\section{La polysémie et l'acquisition des langues secondes ${ }^{1}$}

Cette contribution se situe à l'interface de la linguistique et de la didactique des langues secondes; elle a pour objectif de démontrer les apports d'une analyse de la polysémie verbale à la didactique du français langue seconde. Phénomène omniprésent dans les langues naturelles, la polysémie peut être définie comme une "pluralité de sens liée à une seule forme » (Kleiber, 2005, p. 51). Les multiples sens d'un vocable ne sont pas aléatoires; ils sont liés et partagent des traits communs (p.ex. Victorri \& Fuchs, 1996; Kleiber, 1999; Ravin \& Leacock, 2000). Cependant, les différents sens d'un mot polysémique ne se recoupent pas toujours d'une langue à l'autre, ce qui peut s'avérer problématique pour les apprenants d'une langue seconde (désormais L2).

De manière générale, on s'entend pour dire que la polysémie présente un défi de taille pour les apprenants d'une L2. Par exemple, on note une persistance d'erreurs sémantiques avec les mots polysémiques, même à des étapes avancées de l'acquisition (p. ex. Lennon, 1996). On relève également que les apprenants sont influencés par la polysémie des mots dans leur L1, lorsqu'ils utilisent des mots jugés équivalents dans leur L2 (p.ex. Elston-Güttler \& Williams, 2008; Gullberg, 2009b).

Pour ce qui est des variables qui expliquent la facilité ou la difficulté avec laquelle les différents sens d'un mot polysémique sont acquis, on peut mentionner le caractère concret ou abstrait d'un sens ou encore sa prototypicalité. Selon certaines études (p.ex. Laufer, 1990; Verspoor \& Lowie, 2003), les sens concrets, c'est-à-dire ceux qui font

\footnotetext{
* Corresponding author : leslie.redmond@mun.ca
} 
référence à des objets tangibles, sont plus facilement appris qu'un sens ou un mot abstrait. Qui plus est, plusieurs auteurs parlent de sens noyau ou de sens prototypique, sens qui correspond le plus souvent à un emploi concret. Verspoor et Lowie (2003), par exemple, notent que les sujets de leur étude maîtrisent plus facilement le sens noyau des items auxquels ils sont soumis, surtout lorsque le référent est concret. Par ailleurs, Crossley, Salsbury et McNamara (2010) montrent, pour leur part, que les apprenants s'accrochent à un sens noyau (qui est généralement lié à la fréquence ainsi qu'au caractère concret du référent) et ce n'est que plus tard que les autres sens émergent. Hayashi et Marks (2012), quant à eux, affirment que l'idée du sens prototypique d'un verbe est souvent définie par rapport à la nature sémantique des arguments du verbe, celui-ci prenant un objet concret comme complément direct (p. 160). De plus, la plupart des études qui opérationnalisent une intervention pédagogique permettant de mettre au jour des méthodes d'enseignement qui favorisent l'apprentissage des différents sens des mots polysémiques le font souvent dans le cadre de la linguistique cognitive, cadre dans lequel l'idée d'un sens prototypique est très présente. Par exemple, Verspoor et Lowie (2003) ont montré que connaître le sens concret d'un mot polysémique aide l'apprenant à retenir les sens figurés à long terme. L'étude de Csábi (2004) montre qu'un enseignement fondé sur les principes de la linguistique cognitive, enseignement rendant explicite les liens entre le sens prototypique des verbes hold et keep et leurs autres sens, a un effet positif sur l'apprentissage des différents sens des verbes (p. 248). Les apprenants hongrois qui ont reçu un enseignement explicite sur les liens entre les différents sens ont mieux retenu et utilisé les différents sens des verbes à l'étude que ceux du groupe contrôle, groupe qui a reçu simplement les traductions en hongrois des différentes acceptions des verbes. Par ailleurs, plusieurs chercheurs ont souligné plus précisément la difficulté qu'ont les apprenants à maîtriser des verbes polysémiques (p.ex. go, take, put, make, give) en anglais L2 (p.ex. Csábi, 2004; Hayashi, 2008; Hayashi \& Marks, 2012; Lennon, 1996), et d'autres, moins nombreux, se sont penchés sur les difficultés posées par les verbes polysémiques dans d'autres langues (p.ex. Saji \& Imai, 2013 pour le chinois; Viberg, 2002 pour le suédois). Qui plus est, certains auteurs insistent sur la complexité de la représentation des verbes polysémiques en L2, soulignant le fait qu'il faut prendre en compte non seulement le sens du verbe, mais également le patron syntaxique dans lequel il figure (p.ex. Berthaud \& Antonijevic, 2012; Uçkun, 2012).

Toutefois, bon nombre d'études ne prennent pas en compte l'ensemble des différents sens d'un même mot dans la mesure où elles utilisent, dans leur plan expérimental, un nombre assez élevé de mots polysémiques qui proviennent de catégories syntaxiques différentes (p.ex. Degani \& Tokowicz, 2010; Degani, Tseng \& Tokowicz, 2014; Verpsoor \& Lowie, 2003; Jiang, 2002, 2004). Par ailleurs, les descriptions linguistiques des mots à l'étude sont souvent très hétérogènes (p.ex. Csábi, 2004; Morimoto \& Loewen, 2007; Tyler, 2012; Uçkun, 2012). Dans les études de corpus qui portent pour la plupart sur un nombre assez restreint de mots, les différents sens des mots polysémiques analysés se limitent à ceux produits par les apprenants (p.ex. Altenberg \& Granger, 2001; Bolly, 2007, 2009). Par conséquent, il est difficile d'observer le comportement des apprenants par rapport à l'ensemble des acceptions d'un mot polysémique. Notre étude propose de pallier ces lacunes en étudiant en profondeur la polysémie du verbe prendre dans une perspective linguistique, acquisitionnelle et didactique.

Le verbe prendre est un verbe particulièrement intéressant dans la mesure où il s'agit d'un verbe très fréquent (qui, selon lexique.org, compte 256,16 occurrences par million dans sa forme infinitive) et hautement polysémique. Ainsi, cet article poursuit deux objectifs spécifiques. Notre premier objectif est donc de décrire la polysémie du verbe prendre au moyen d'une analyse en sémantique lexicale dans une approche cognitive. Cette analyse nous permettra également d'émettre des hypothèses sur les différences entre le verbe prendre et ses équivalents en anglais. Notre deuxième objectif est d'évaluer l'incidence de la polysémie du verbe prendre sur les connaissances qu'ont les apprenants de ce verbe et d'isoler les différentes acceptions de prendre qui s'avèrent problématiques pour les apprenants du français L2. Dans ce qui suit, nous présenterons l'analyse sémantique du verbe prendre que nous proposons, puis l'étude empirique qui en découle auprès d'apprenants du français L2.

\section{Analyse de la polysémie du verbe prendre}

La polysémie du verbe prendre a déjà fait l'objet d'un certain nombre d'études, qui poursuivaient des objectifs différents (p.ex. El Guedri, 2011; Manes Gallo \& Rouault, 2010; Paillard, 2001; Peeters \& Eiszele, 1993; Sénéchal, 2012). Picoche (1991) a proposé, à notre connaissance, l'analyse du verbe prendre la plus exhaustive. Cette analyse s'appuie sur des critères de classement sémantiques; les critères syntaxiques sont au deuxième plan (p. 161). Cette étude constitue, d'une certaine manière, notre point de départ dans la mesure où elle met en exergue les liens entre les différents sens du verbe prendre qui, de prime abord, semblent être très différents les uns des autres. Ainsi nous nous proposons de tenter d'unifier l'ensemble de ces acceptions dans une approche monosémique de la polysémie (voir Ruhl, 1989). ${ }^{2}$

Afin d'analyser les acceptions prédicatives du verbe prendre, nous nous sommes fondées sur des données lexicales issues de plusieurs dictionnaires ${ }^{3}$ de référence et d'un dictionnaire du lexique verbal informatisé (Les Verbes français). Chaque acception a été codée selon plusieurs critères : le type sémantique des noms du co-texte ${ }^{4}$, le patron syntaxique (nombre et type syntaxique des arguments internes), les synonymes du verbe ${ }^{5}$ ainsi que les traductions en anglais du verbe prendre validées par des jugements de grammaticalité auprès de locuteurs natifs de l'anglais. Les verbes prendre et take partagent un certain nombre de leurs acceptions, mais ils différent dans d'autres cas pour lesquels une acception du verbe prendre en français se réalise par un verbe tel que get, have, catch ou encore par le verbe take suivi d'une particule.

Ainsi, pour rendre compte de la polysémie du verbe prendre, nous proposons un noyau de sens abstrait présent 
dans l'ensemble des acceptions prédicatives du verbe et se déployant de manière différente selon le sens actualisé. Ce noyau est composé de trois parties : (1) une orientation du sujet vers l'objet, (2) un contact et (3) une orientation de l'objet vers le sujet. Nous proposons également que seul un élément du noyau puisse être fenêtré pour chaque acception. Nous avons eu recours au concept du fenêtrage de l'attention de Talmy (2000), processus cognitif selon lequel l'attention d'un locuteur est focalisée sur une portion ou une autre d'un événement, au détriment des autres parties reléguées au deuxième plan, ou écartées (p. 28). La partie mise en évidence est fenêtrée. Pour qu'un élément soit fenêtré, il faut qu'il devienne saillant de par les formes linguistiques présentes; c'est en effet l'inclusion des informations linguistiques explicites relatives à une partie de l'événement qui permet la mise en évidence ou le fenêtrage de celle-ci (Talmy, 2000, p. 258). Le fait qu'une partie d'une scène est fenêtrée n'estompe pas les autres parties écartées; elles sont simplement moins saillantes que celle qui fait l'objet d'un fenêtrage de l'attention.

\subsection{Le contact est fenêtré}

Les premières acceptions que nous présentons sont celles pour lesquelles le contact est fenêtré. Le contact peut se faire par saisie ou par localisation. Dans les exemples (1) et (2), le contact se fait par saisie.

(1) L'enfant prend un livre sur l'étagère. (The child takes a book from the shelf.)

(2) Marie prend sa fille par la main. (Marie takes her daughter by the hand.)

Dans ces acceptions, l'orientation du sujet vers l'objet correspond au fait que le sujet tend sa main pour saisir l'objet. Le contact entre le sujet et l'objet est physique et manuel. À la suite du contact, le sujet possède l'objet, ce qui présuppose que l'objet ait été déplacé de son lieu d'origine jusqu'au sujet: il s'agit là de la manifestation de l'orientation de l'objet vers le sujet. Dans ces acceptions, les orientations que ce soit celle du sujet vers l'objet ou celle de l'objet vers le sujet ne sont pas saillantes.

Le contact par saisie, qui est manuel et physique, n'est pas limité aux objets physiques qui sont des artefacts. Il vaut également pour les entités physiques qui sont des matières qui peuvent être des solides ou des substances, comme l'exemple en (3) nous le montre.

(3) Il prend ses médicaments. (He takes his medication.)

L'acception peut donc acquérir une autre valeur si on met l'accent sur l'aspect ingérable de l'entité complément direct. Dans ce cas, le contact par saisie et l'orientation de l'objet vers le sujet qui en découle correspondent à l'ingestion.

Le contact peut être également abstrait comme dans le cas de l'exemple en (4).

(4) Elle a pris la citation dans un livre. (She took the quote from a book.)

Ici, le sujet dirige son esprit vers l'entité abstraite en question; il s'agit là de l'orientation du sujet vers l'objet. Quant au contact, il se fait toujours par saisie, mais il s'agit d'une saisie cognitive. L'orientation de l'objet vers le sujet correspond à un chemin possessionnel qui va du lieu source de l'objet vers le sujet. Dans les exemples en (5), (6), et (7), le contact est toujours fenêtré, mais il se fait par localisation. L'espace est lexicalisé par le syntagme nominal argument.

(5) Les Français ont pris l'Alsace. (The French took Alsace.)

(6) Elle prend souvent la voiture. (She often takes the car.)

(7) On a pris l'autoroute pour aller à Toronto. (We took the highway to go to Toronto.)

L'orientation du sujet vers l'objet se manifeste par le fait que le sujet se rend à l'espace dénoté par le complément direct. Finalement, l'orientation de l'objet vers le sujet se manifeste par l'appropriation ou l'utilisation que fait le sujet de cet espace.

La contrepartie abstraite de cette acception correspond aux cas pour lesquels le complément direct n'est plus un espace physique, mais plutôt un espace de temps comme dans les exemples en (8) et (9).

(8) Elle a pris une heure pour faire ce travail. (She took an hour to do that job.)

(9) Ils prennent leurs vacances. (They take their vacation.)

L'expression du noyau de sens se fait de la manière suivante. Le sujet établit une relation avec le temps dénoté par le complément direct - c'est la manifestation de l'orientation du sujet vers l'objet. Le contact par localisation est abstrait et se manifeste par le fait que le sujet dispose d'un créneau de temps lexicalisé par le complément direct. L'orientation de l'objet vers le sujet se manifeste dans l'utilisation que fait le sujet de l'espace de temps. 


\subsection{L'orientation du sujet vers l'objet est fenêtrée}

Passons maintenant aux cas pour lesquels l'orientation du sujet vers l'objet est fenêtrée (orientation 1). Le contact et l'orientation de l'objet vers le sujet sont moins saillants. Il s'agit des exemples en (10), (11), (12) et (13).

(10) Elle a pris un livre à la bibliothèque. (She got/took out a book from the library.)

(11) Il a pris du pain à la boulangerie/chez le boulanger. (He got bread at the bakery/at the baker's.)

(12) Marie prend sa fille à 15 heures. (Marie gets/picks up her daughter at 3pm.)

(13) Jean prend sa collègue à l'aéroport. (John gets/picks up his colleague at the airport.)

Puisque l'orientation du sujet vers l'objet est fenêtrée, elle devient saillante et diffère ainsi de la manifestation déjà décrite pour les cas où le contact est fenêtré. Il ne s'agit pas du sujet qui tend la main, comme dans l'exemple Pierre prend un livre sur l'étagère, mais du sujet qui se déplace vers le lieu où il va retrouver l'objet. La saillance de cette orientation se manifeste par l'ajout obligatoire de repères spatiaux ou temporels dans le co-texte (des syntagmes prépositionnels, le plus souvent), repères qui permettent de mettre en évidence le rapport existant entre le sujet et le lieu où se trouve l'entité complément direct. Dans le co-texte du verbe prendre, le lieu peut être lexicalisé explicitement dans le syntagme prépositionnel (p.ex. à la bibliothèque); on peut également l'inférer à partir d'un syntagme prépositionnel qui fait référence à un lieu non intrinsèque (p.ex. chez le boulanger) ou encore, il peut être un espace de temps (p.ex. à 15 heures). Le contact se manifeste de deux façons : par saisie lorsque le complément direct est prenable par les mains comme dans les exemples (10) et (11) ou par localisation lorsqu'il ne l'est pas, comme dans les exemples (12) et (13). L'orientation de l'objet vers le sujet, quant à elle, se manifeste par un chemin possessionnel qui va du lieu source de l'objet vers le sujet ((10) et (11)) ou encore pour les cas de contact par localisation, par le partage d'un espace commun. Ainsi, le déplacement du sujet vers l'objet estompe d'une certaine façon le contact par saisie ou localisation ainsi que l'orientation de l'objet vers le sujet qui en découle.

Passons maintenant aux acceptions pour lesquelles l'orientation de l'objet vers le sujet est fenêtrée.

\subsection{L'orientation de l'objet vers le sujet est fenêtrée}

Lorsque l'orientation de l'objet vers le sujet est fenêtrée (orientation 2), le contact peut également se faire par saisie ou par localisation. Présentons tout d'abord les acceptions pour lesquelles le contact se fait par saisie. Rappelons que la manifestation prototypique de cette orientation est le mouvement de l'objet sur un chemin possessionnel qui va du lieu source de l'objet jusqu'au sujet. Nous proposons que, lorsqu'elle est fenêtrée, cette orientation se manifeste de trois façons différentes selon les éléments du co-texte : la perte de contrôle, la localisation finale dans une entité liée au sujet et l'utilisation. Pour ce qui est des autres parties du noyau de sens, l'orientation du sujet vers l'objet et le contact par saisie, elles se manifestent différemment selon les types sémantiques des éléments du co-texte (voir les différentes manifestations de l'orientation 1 et du contact par saisie décrites dans les parties 2.1 et 2.2). La première manifestation de l'orientation 2 est la perte de contrôle, comme dans les exemples (14) et (15).

(14) La police a pris le voleur. (The police caught/got the thief.)

(15) L'orage l'a pris en pleine campagne. (He got caught in the storm.)

L'élément complément direct, contre sa volonté, passe sous l'emprise du sujet. L'orientation 2 correspond ainsi à une perte de contrôle. Le sujet peut être une entité vivante (14) ou une entité abstraite (15). Pour le cas des entités abstraites (sujets), l'élément complément direct passe donc d'un état où il ne subit pas les effets du sujet à un état où il les subit. Ensuite, l'orientation de l'objet vers le sujet peut se manifester comme la localisation finale de l'élément complément direct avec le sujet. Dans ces cas, le co-texte du verbe prendre doit inclure un syntagme prépositionnel représentant un lieu relié au sujet (intrinsèque, non intrinsèque ou par métonymie).

(16) Max a pris les pommes dans son panier. (Max put the apples in his basket.)

(17) Elle a pris une citation de Talmy dans son livre. (She put one of Talmy's quotes in her book.)

(18) L'enseignant a pris deux nouveaux étudiants dans sa classe. (The teacher accepted two new students in his class.)

(19) Marie a pris une amie chez elle. (Marie took in a friend.)

Par ailleurs, certaines entités ont intrinsèquement des propriétés associées à un lieu tel que l'exemple suivant nous le montre.

(20) Marie a pris un colocataire. (Marie got a roommate.)

Dans ce cas, sont imbriqués dans l'élément complément direct non seulement les traits relevant de l'entité vivante mais aussi ceux du lieu relié au sujet. 
Finalement, pour les cas où l'orientation 2 se manifeste comme l'utilisation de l'entité complément direct, une manière d'utiliser l'élément est lexicalisée dans un syntagme prépositionnel introduit par comme. Qui plus est, le nom complément direct a, dans tous les cas, les mêmes traits sémantiques que le nom introduit par la préposition comme.

(21) Il a pris la serviette comme couverture. (He used the towel as a blanket.)

(22) On a pris Sarah comme secrétaire. (We hired Sarah as a secretary.)

Enfin, il s'agit de discuter des cas pour lesquels l'orientation 2 est fenêtrée avec un contact par localisation. Il s'agit là d'acceptions intransitives. L'ensemble de ces acceptions évoque un changement d'état du sujet, changement dû au type sémantique du sujet - lui-même composé de plusieurs éléments, tels que les exemples en (23) et (24) nous le montrent.

(23) La mayonnaise a pris. (The mayonnaise thickened.)

(24) Le mortier prend. (The mortar set.)

Ici, l'orientation 1 correspond à la causation. Ensuite, le sujet a des propriétés inhérentes, qui, par un contact par localisation, lui permettent de changer d'état, comme le mortier qui passe d'un état liquide à un état solide. L'orientation 2, pour sa part, peut être décrite comme étant un processus qui résulte en la réussite du sujet (l'entité complète) dans la mesure où le sujet passe de plusieurs composants à une entité complète.

Finalement, prenons les exemples en (25) et (26), exemples pour lesquels le sujet est une entité abstraite.

(25) Notre nouveau produit n'a pris qu'en province. (This product only caught on in the regions.)

(26) Cette mode n'a pas pris. (This style didn't catch on.)

Pour ce qui est des entités abstraites, elles sont constituées de plusieurs composants, eux aussi abstraits, qui peuvent rentrer en contact (le contact par localisation) afin de produire un effet (l'orientation vers le sujet).

\subsection{Synthèse de l'analyse sémantique}

Le but de notre analyse sémantique était d'unifier l'ensemble des acceptions prédicatives du verbe prendre sous un même noyau de sens abstrait. Nous avons proposé que ce noyau de sens soit tripartite et intègre une orientation du sujet vers l'objet (orientation 1), un contact et une orientation de l'objet vers le sujet (orientation 2). Nous avons également effectué une démarche comparative avec l'anglais (validée par des données dictionnairiques ainsi que des jugements de grammaticalité de locuteurs natifs de l'anglais) nous permettant d'émettre des hypothèses sur les différences et les similarités entre les verbes prendre et take. Ces verbes semblent partager le concept du contact, qu'il se fasse par saisie ou par localisation. En effet, ce sont les acceptions pour lesquelles le contact est fenêtré qui sont communes aux deux verbes. En revanche, lorsque l'une ou l'autre des orientations est fenêtrée, take ne peut pas être utilisé comme équivalent de prendre. Le fenêtrage des différentes parties du noyau de sens permet donc de distinguer les acceptions du verbe prendre qui se traduisent par take en anglais et celles qui se traduisent par un verbe autre que take (ou take suivi d'une particule).

\section{3 Étude empirique}

Le second objectif de ce travail est d'opérationnaliser notre analyse sémantique dans une étude empirique auprès d'apprenants du français L2. Il s'agit de mettre au jour les acceptions du verbe prendre qui sont les plus facilement acquises par ces apprenants et d'évaluer les facteurs linguistiques sous-tendant cette acquisition. Dans ce qui suit, nous exposerons la méthode de l'étude empirique, puis les résultats de celle-ci.

\subsection{Méthode}

Afin de participer à l'étude, les sujets devaient être inscrits à un programme universitaire ou collégial qui comprend des cours de français L2. 191 participants ont pris part à l'étude : 109 femmes et 82 hommes dont l'âge moyen est de 22,14 ans. Les participants appartiennent à deux groupes linguistiques distincts : les anglophones (leur langue maternelle est l'anglais $n=118$ ) et les allophones (leur langue maternelle est une langue autre que l'anglais $n=73$ ). ${ }^{6}$ Dans les écrits méthodologiques sur l'influence translangagière, on insiste sur l'idée que la vérification de l'influence translangagière doit reposer sur différents critères, l'un de ces critères étant l'hétérogénéité intergroupe (Jarvis \& Pavlenko, 2008, p. 45). On peut confirmer l'hétérogénéité intergroupe «[...] lorsqu'un groupe d'apprenants comparables d'une L2 commune qui ont différentes langues maternelles diffèrent dans leur performance dans la L2 » (notre traduction de Jarvis, 2000, p. 254 : [...] when comparable learners of a common L2 who speak different L1s diverge in their Il [interlanguage] performance).

L'expérience (durée totale: 1h) s'est déroulée en salle de classe en présence de l'expérimentateur. Les 
participants ont effectué plusieurs tâches dans l'ordre suivant. D'abord, ils ont effectué une tâche de production guidée lors de laquelle ils devaient écrire six phrases comportant le verbe prendre. Ensuite, ils ont effectué une tâche lacunaire ayant pour but de mesurer leur niveau de compétence langagière en français L2 ( $\min =7 / 30$; $\max =26 / 30$; moyenne=17,85). Il nous a fallu vérifier qu'il n'y avait pas de lien entre nos deux variables indépendantes qui relèvent des caractéristiques des participants : le groupe linguistique des participants (anglophone ou allophone) et le score au test lacunaire, notre mesure du niveau en français L2. Les résultats d'un ANOVA confirment l'absence de ce lien [F $(1,189)=0,42, \mathrm{p}=\mathrm{ns}]$.

Puis, les participants ont effectué une tâche de jugement de grammaticalité, tâche dans laquelle ils devaient lire un court contexte en français et choisir la ou les phrases pouvant suivre ce contexte. Pour chaque item (voir exemple (27)), il y a une phrase avec un emploi correct du verbe prendre (27-a), une phrase avec un synonyme correct du verbe prendre dans le contexte $(27-\mathrm{c})$ et une phrase qui comporte une erreur (p.ex. un synonyme qui ne répond pas au contexte, un verbe asémantique dans le contexte ou encore un verbe qui encode une orientation qui ne répond pas au contexte (27-b)).

\section{(27) Pierre fait souvent ses commissions après le travail.}

a. Tous les soirs en rentrant du travail, Pierre prend du pain à la boulangerie.

b. Tous les soirs en rentrant du travail, Pierre apporte du pain à la boulangerie.

c. Tous les soirs en rentrant du travail, Pierre achète du pain à la boulangerie.

Le test comportait 24 items pour lesquels les différents paramètres de notre analyse sémantique (le type de contact et le fenêtrage) ont été manipulés. ${ }^{7}$ Enfin, un questionnaire de données sociodémographiques est venu clore l'expérience.

\subsection{Résultats}

Afin d'analyser les résultats, nous avons eu recours à des analyses de régression logistique, analyses dans lesquelles le coefficient de régression s'exprime au moyen d'un rapport de chances qui correspond à «[...] la probabilité d'occurrence d'un événement (e.g. la probabilité de voter) sur la probabilité de non-occurrence (e.g. la probabilité de ne pas voter) » (Bressoux, 2008, p. 229). Nous présenterons d'abord les résultats de la tâche de production écrite, ensuite ceux de la tâche de jugement de grammaticalité. Dans la présentation des tableaux, les variables fenêtrage et type de contact correspondent aux catégories mises au jour par notre analyse sémantique. La variable fenêtrage se décline en trois modalités : l'orientation du sujet vers l'objet est fenêtrée (orientation 1), le contact est fenêtré (contact) et l'orientation de l'objet vers le sujet est fenêtrée (orientation 2). La variable type de contact, quant à elle, comprend deux modalités : le contact peut se réaliser par saisie ou par localisation.

\subsubsection{Tâche de production écrite}

Analysons d'abord les phrases produites lors de la tâche de production écrite. Nous analysons un total de 660 phrases qui contiennent une acception prédicative du verbe prendre. ${ }^{8}$ Les tableaux $1 \mathrm{a}, 1 \mathrm{~b}$ et $1 \mathrm{c}$ font état des données descriptives.

Tableau 1a: Données descriptives pour tous les participants

\begin{tabular}{|c|c|c|c|c|c|c|c|c|}
\hline Fenêtrage & Orientat & & Contact & & Orientati & & Total & \\
\hline Type de contact & Effectif & $\%(\mathrm{ET})$ & Effectif & $\%(\mathrm{ET})$ & Effectif & $\%(\mathrm{ET})$ & Effectif & $\%(\mathrm{ET})$ \\
\hline Par saisie & 2 & $\begin{array}{l}28,6 \\
(0,18)\end{array}$ & 362 & $\begin{array}{l}57,2 \\
(0,02)\end{array}$ & 19 & $\begin{array}{l}95 \\
(0,05)\end{array}$ & 383 & $\begin{array}{l}58,0 \\
(0,02)\end{array}$ \\
\hline Par localisation & 5 & $\begin{array}{l}71,4 \\
(0,18)\end{array}$ & 271 & $\begin{array}{l}42,8 \\
(0,02)\end{array}$ & 1 & $\begin{array}{l}5,0 \\
(0,05)\end{array}$ & 277 & $\begin{array}{l}42,0 \\
(0,02)\end{array}$ \\
\hline Total & 7 & $\begin{array}{l}1,1 \\
(0,004)\end{array}$ & 633 & $\begin{array}{l}96,0 \\
(0,01)\end{array}$ & 20 & $\begin{array}{l}3,0 \\
(0,01)\end{array}$ & 660 & 100 \\
\hline
\end{tabular}


Tableau 1b: Données descriptives pour le groupe anglophone

\begin{tabular}{|c|c|c|c|c|c|c|c|c|}
\hline Fenêtrage & Orientat & & Contact & & Orientat & & Total & \\
\hline Type de contact & Effectif & $\%(\mathrm{ET})$ & Effectif & $\%(\mathrm{ET})$ & Effectif & $\%(\mathrm{ET})$ & Effectif & $\%(\mathrm{ET})$ \\
\hline Par saisie & 2 & $\begin{array}{l}50,0 \\
(0,03)\end{array}$ & 263 & $\begin{array}{l}58,0 \\
(0,02)\end{array}$ & 14 & 100 & 279 & $\begin{array}{l}59,1 \\
(0,02)\end{array}$ \\
\hline Par localisation & 2 & $\begin{array}{l}50,0 \\
(0,03)\end{array}$ & 191 & $\begin{array}{l}43,0 \\
(0,02)\end{array}$ & 0 & 0 & 193 & $\begin{array}{l}40,8 \\
(0,02)\end{array}$ \\
\hline Total & 4 & $\begin{array}{l}1,0 \\
(0,004)\end{array}$ & 454 & $\begin{array}{l}96,2 \\
(0,01)\end{array}$ & 14 & $\begin{array}{l}3,0 \\
(0,01)\end{array}$ & 472 & 100 \\
\hline
\end{tabular}

Tableau 1c: Données descriptives pour le groupe allophone

\begin{tabular}{|c|c|c|c|c|c|c|c|c|}
\hline Fenêtrage & Orientati & & Contact & & Orientat & & Total & \\
\hline Type de contact & Effectif & $\%(\mathrm{ET})$ & Effectif & $\%(\mathrm{ET})$ & Effectif & $\%(\mathrm{ET})$ & Effectif & $\%(\mathrm{ET})$ \\
\hline Par saisie & 0 & 0 & 99 & $\begin{array}{l}55,3 \\
(0,04)\end{array}$ & 5 & $\begin{array}{l}83,3 \\
(0,17)\end{array}$ & 104 & $\begin{array}{l}55,3 \\
(0,04)\end{array}$ \\
\hline Par localisation & 3 & 100 & 80 & $\begin{array}{l}44,7 \% \\
(0,04)\end{array}$ & 1 & $\begin{array}{l}16,7 \\
(0,17)\end{array}$ & 84 & $\begin{array}{l}44,7 \\
(0,04)\end{array}$ \\
\hline Total & 3 & $\begin{array}{l}1,6 \\
(0,01)\end{array}$ & 179 & $\begin{array}{l}95,2 \\
(0,02)\end{array}$ & 6 & $\begin{array}{l}3,2 \\
(0,01)\end{array}$ & 188 & 100 \\
\hline
\end{tabular}

Nous avons d'abord effectué des analyses de khi carré afin de vérifier s'il y avait un lien entre le groupe linguistique des participants et les variables issues de l'analyse sémantique (la partie du noyau de sens fenêtrée et le type de contact). Aucune des deux analyses n'est significative; les participants ont massivement produit des phrases dans lesquelles le contact est fenêtré (96,0\% des acceptions prédicatives produites); les deux types de contact étaient représentés à peu près également du point de vue statistique : contact par saisie $(52,8 \%)$ et contact par localisation $(47,2$ $\%)$. De plus, les données descriptives ne montrent que très peu de différences entre les apprenants anglophones et allophones.

Puis, nous avons vérifié s'il existait un lien entre le type de contact actualisé dans la phrase produite en fonction de la partie du noyau de sens fenêtrée, le groupe linguistique du participant et son niveau de compétence langagière en français L2 (tableau 2).

Tableau 2 : Régression logistique sur les acceptions prédicatives à la tâche de production écrite

\begin{tabular}{llll}
\hline & Rapport de chances & Erreur type & Sig. \\
\hline Constante & 1,50 & 0,58 & 0,288 \\
Niveau & 0,99 & 0,02 & 0,840 \\
$\begin{array}{l}\text { Langue } \\
\text { (Anglophone-réf.) }\end{array}$ & 0,85 & 0,15 & 0,372
\end{tabular}

\section{Fenêtrage}

(orientation 1-réf.)

$\begin{array}{llll}\text {-Contact } & 3,28 & 2,76 & 0,158\end{array}$

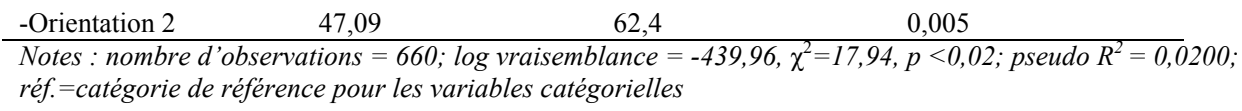


Les résultats montrent que ce n'est que pour les acceptions pour lesquelles l'orientation 2 est fenêtrée que la distinction entre le contact par saisie et le contact par localisation est pertinente. Les variables liées au groupe linguistique du participant ainsi qu'à son niveau de compétence langagière ne sont pas significatives.

Passons à l'analyse des données issues de la tâche de jugement.

\subsubsection{Tâche de jugement}

Afin d'analyser la tâche de jugement grammaticalité, nous avons effectué une analyse de régression logistique sur les réponses données. Rappelons que les participants avaient comme consigne de choisir toutes les phrases qui pouvaient suivre le contexte. Nous considérons comme bonnes réponses les cas où l'apprenant choisit le verbe prendre (p.ex. 27a), un synonyme (p.ex. 27-c) ou encore, les deux. Les mauvaises réponses, quant à elles, comportent une erreur (p.ex. 27-b). 17,8 \% des réponses fournies étaient des erreurs. Dans cet article, nous n'analysons que les bonnes réponses en opposant le choix du verbe prendre (avec ou sans synonyme) à celui du synonyme seul. Les tableaux suivants ( $3 \mathrm{a}, 3 \mathrm{~b}$ et 3c) présentent les données descriptives en fonction des variables linguistiques ainsi que du groupe linguistique auquel appartient le participant.

Tableau 3a: Données descriptives pour tous les participants

\begin{tabular}{|c|c|c|c|c|c|c|c|c|}
\hline Fenêtrage & Orientati & & Contact & & Orientat & & Total & \\
\hline Type de contact & Effectif & $\%(\mathrm{ET})$ & Effectif & $\%(\mathrm{ET})$ & Effectif & $\%(\mathrm{ET})$ & Effectif & $\%(\mathrm{ET})$ \\
\hline Par saisie & 141 & $\begin{array}{l}45,2 \\
(0,03)\end{array}$ & 536 & $\begin{array}{l}64,5 \\
(0,02)\end{array}$ & 461 & $\begin{array}{l}36,6 \\
(0,01)\end{array}$ & 1138 & $\begin{array}{l}47,4 \\
(0,01)\end{array}$ \\
\hline Par localisation & 94 & $\begin{array}{l}35,3 \\
(0,03)\end{array}$ & 390 & $\begin{array}{l}84,6 \\
(0,02)\end{array}$ & 170 & $\begin{array}{l}31,7 \\
(0,02)\end{array}$ & 654 & $\begin{array}{l}51,2 \\
(0,01)\end{array}$ \\
\hline Total & 235 & $\begin{array}{l}40,7 \\
(0,02)\end{array}$ & 926 & $\begin{array}{l}71,7 \\
(0,01)\end{array}$ & 631 & $\begin{array}{l}35,1 \\
(0,01)\end{array}$ & 1792 & $\begin{array}{l}48,9 \\
(0,01)\end{array}$ \\
\hline
\end{tabular}

Tableau 3b: Données descriptives pour le groupe anglophone

\begin{tabular}{|c|c|c|c|c|c|c|c|c|}
\hline Fenêtrage & Orientat & & Contact & & Orientat & & Total & \\
\hline Type de contact & Effectif & $\%(\mathrm{ET})$ & Effectif & $\%(\mathrm{ET})$ & Effectif & $\%(\mathrm{ET})$ & Effectif & $\%(\mathrm{ET})$ \\
\hline Par saisie & 81 & $\begin{array}{l}42,6 \\
(0,04)\end{array}$ & 345 & $\begin{array}{l}67,0 \\
(0,02)\end{array}$ & 270 & $\begin{array}{l}35,2 \\
(0,02)\end{array}$ & 696 & 47,3 \\
\hline Par localisation & 53 & $\begin{array}{l}30,5 \\
(0,03)\end{array}$ & 238 & $\begin{array}{l}84,4 \\
(0,02)\end{array}$ & 101 & $\begin{array}{l}30,1 \\
(0,02)\end{array}$ & 392 & 49,5 \\
\hline Total & 134 & $\begin{array}{l}36,8 \\
(0,03)\end{array}$ & 583 & $\begin{array}{l}73,1 \\
(0,02)\end{array}$ & 371 & $\begin{array}{l}33,6 \\
(0,01)\end{array}$ & 1088 & $\begin{array}{l}48,0 \\
(0,01)\end{array}$ \\
\hline
\end{tabular}

Tableau 3c: Données descriptives pour le groupe allophone

\begin{tabular}{|c|c|c|c|c|c|c|c|c|}
\hline Fenêtrage & Orientat & & Contact & & Orientat & & Total & \\
\hline Type de contact & Effectif & $\%(\mathrm{ET})$ & Effectif & $\%(\mathrm{ET})$ & Effectif & $\%(\mathrm{ET})$ & Effectif & $\%(\mathrm{ET})$ \\
\hline Par saisie & 60 & $\begin{array}{l}49,1 \\
(0,05)\end{array}$ & 191 & $\begin{array}{l}60,6 \\
(0,03)\end{array}$ & 191 & $\begin{array}{l}39,0 \\
(0,02)\end{array}$ & 442 & $\begin{array}{l}47,6 \\
(0,02)\end{array}$ \\
\hline Par localisation & 41 & $\begin{array}{l}44,6 \\
(0,05)\end{array}$ & 152 & $\begin{array}{l}85,0 \\
(0,03)\end{array}$ & 69 & $\begin{array}{l}34,5 \\
(0,03)\end{array}$ & 262 & $\begin{array}{l}55,6 \\
(0,02)\end{array}$ \\
\hline Total & 101 & $\begin{array}{l}47,2 \\
(0,03)\end{array}$ & 343 & $\begin{array}{l}69,4 \\
(0,02)\end{array}$ & 260 & $\begin{array}{l}37,6 \\
(0,02)\end{array}$ & 704 & $\begin{array}{l}50,3 \\
(0,01)\end{array}$ \\
\hline
\end{tabular}

Le tableau 4 présente les données issues de l'analyse de régression logistique. Il s'agit d'un plan d'analyse factoriel comportant quatre effets principaux (le niveau (variable contrôle), le groupe linguistique du participant, la partie du noyau de sens fenêtrée et le type de contact) ainsi que leurs interactions. 
Tableau 4: Régression logistique sur le choix du verbe prendre (avec ou sans synonyme)

\begin{tabular}{|c|c|c|c|}
\hline & Rapport de chances & Erreur type & Sig. \\
\hline Constante & 1,18 & 0,22 & 0,374 \\
\hline Niveau & 0,97 & 0,01 & 0,001 \\
\hline $\begin{array}{l}\text { Langue } \\
\text { (Allophone-réf.) }\end{array}$ & 0,82 & 0,10 & 0,116 \\
\hline \multicolumn{4}{|l|}{$\begin{array}{l}\text { Fenêtrage } \\
\text { (Orientation 2-réf.) }\end{array}$} \\
\hline $\begin{array}{l}\text {-Contact } \\
\text {-Orientation } 1\end{array}$ & $\begin{array}{l}2,44 \\
1,54\end{array}$ & $\begin{array}{l}0,37 \\
0,32\end{array}$ & $\begin{array}{l}0,001 \\
0,04\end{array}$ \\
\hline $\begin{array}{l}\text { Type de contact } \\
\text { (par saisie-réf.) }\end{array}$ & 0,83 & 0,15 & 0,317 \\
\hline \multicolumn{4}{|l|}{ Langue \# fenêtrage } \\
\hline -Anglophone \# contact & 1,61 & 0,32 & 0,02 \\
\hline -Anglophone \# orientation 1 & 0,93 & 0,25 & 0,778 \\
\hline \multicolumn{4}{|l|}{ Langue \# type de contact } \\
\hline -Anglophone \# par localisation & 0,99 & 0,23 & 0,963 \\
\hline \multicolumn{4}{|l|}{ Fenêtrage \# type de contact } \\
\hline -Contact \# par localisation & 5,16 & 1,60 & 0,001 \\
\hline -Orientation 1 \# par localisation & 1,00 & 0,33 & 0,983 \\
\hline \multicolumn{4}{|l|}{$\begin{array}{l}\text { Langue \# fenêtrage \# type de } \\
\text { contact }\end{array}$} \\
\hline $\begin{array}{l}\text {-Anglophone \# contact \# par } \\
\text { localisation }\end{array}$ & 0,63 & 0,24 & 0,231 \\
\hline $\begin{array}{l}\text {-Anglophone \# orientation } 1 \text { \# } \\
\text { par localisation }\end{array}$ & 0,72 & 0,31 & 0,442 \\
\hline
\end{tabular}

Notes : nombre d'observations $=3535 ; \log$ vraisemblance $=-2181,72, \chi^{2}=535,43, p<0,001 ;$ pseudo $R^{2}=0,1093$; réf. $=$ catégorie de référence pour les variables catégorielles

Nous avons tout d'abord contrôlé la variation due au niveau de compétence langagière des participants. En effet, un moins bon score au test lacunaire correspond à plus de chances de choisir le verbe prendre (avec ou sans synonyme) par rapport à un synonyme seul, toutes choses étant égales par ailleurs $(0,97, \mathrm{p}<0,001)$. Pour ce qui est des effets principaux, seul l'effet du fenêtrage permet de prédire le choix du verbe prendre (avec ou sans synonyme) contre un synonyme seul à la tâche de jugement, montrant par là même le pouvoir prédictif de notre analyse sémantique. Les participants ont 2,44 ( $<<0,001)$ fois plus de chances de choisir le verbe prendre pour les acceptions pour lesquelles le contact est fenêtré que pour celles pour lesquelles l'orientation 2 est fenêtrée et $3,91(\mathrm{p}<0,01)$ fois plus de chances que pour celles pour lesquelles l'orientation 1 est fenêtrée. Enfin, ils ont 1,54 $(\mathrm{p}<0,04)$ plus de chances de choisir le verbe prendre (avec ou sans synonyme) pour les acceptions pour lesquelles l'orientation 1 est fenêtrée que pour celles pour lesquelles l'orientation 2 est fenêtrée.

Examinons maintenant les effets d'interaction. Deux interactions à deux facteurs sont significatives : celle entre les variables langue et fenêtrage $\left(\chi^{2}(2)=7,04, p<0,03\right)$ et celle entre les variables fenêtrage et type de contact $\left(\chi^{2}\right.$ $(2)=31,19, p<0,001)$. Autrement dit, au-delà de l'effet de toutes les autres variables, les participants anglophones ont 1,61 fois plus de chances de choisir le verbe prendre (avec ou sans synonyme) lorsqu'il s'agit d'une acception pour laquelle le contact est fenêtrée. De plus, tous les participants ont 5,16 ( $<<0,001)$ fois plus de chances de choisir le verbe prendre (avec ou sans synonyme) pour les acceptions pour lesquelles le contact est fenêtrée et se fait par localisation. 


\section{Discussion des résultats}

Dans la discussion, nous reprenons les résultats de notre étude à la lumière de ceux des études en acquisition et en didactique des langues secondes portant sur la maîtrise des différents sens des mots polysémiques en L2. Nous parlons du rôle de la langue maternelle (4.1), de la prototypicalité (4.2) ainsi que du caractère concret ou abstrait d'une acception (4.3).

\subsection{Le rôle de la langue maternelle}

De manière générale, les auteurs s'entendent sur le fait que les discordances entre les langues sur le plan lexicosémantique constituent une pierre d'achoppement pour les apprenants d'une L2 et que la restructuration sémantique qui en découle est une tâche ardue, confirmant ainsi la présence de l'influence translangagière dans l'acquisition des mots polysémiques dans une L2 (p. ex. Degani \& Tokowicz, 2010; Gullberg, 2009a; Ijaz, 1986; Jiang, 2002, 2004; Morimoto \& Loewen, 2007).

À la tâche de production écrite, aucune différence liée au groupe linguistique des participants n'a été observée. Cependant, à la tâche de jugement d'acceptabilité, l'interaction entre les variables langue et fenêtrage s'est avérée significative : les anglophones ont 1,61 fois plus de chances $(\mathrm{p}<0,02)$ de choisir le verbe prendre (avec ou sans synonyme) lorsque le contact est fenêtré. Rappelons que ce sont ces acceptions qui sont communes aux verbes take et prendre, contrairement à celles pour lesquelles l'une des orientations est fenêtrée. Nous avons interprété la différence entre les deux groupes comme étant un indice de l'influence translangagière chez les apprenants anglophones; le groupe des allophones constituant une sorte de groupe contrôle (p.ex. Jarvis, 2000; Jarvis \& Pavlenko, 2008). ${ }^{9}$

Dans notre analyse sémantique de la polysémie du verbe prendre, la variable liée au fenêtrage de l'attention permet de différencier les acceptions du verbe prendre partagées avec le verbe take de celles pour lesquelles l'acception $\mathrm{du}$ verbe prendre en français se traduit par un verbe autre que take en anglais. Notons que l'interaction entre les variables langue et type de contact n'est pas significative. Ainsi, nous pouvons arguer que puisque les différents types de contact permettent de rendre compte des variations de sens du verbe prendre en français, mais non des différences entre les verbes prendre et take, le comportement que nous avons observé vis-à-vis de la variable fenêtrage est bel et bien un comportement qui s'explique par l'influence translangagière chez les apprenants anglophones. Comment rendre compte de la différence entre les deux tâches en ce qui a trait à l'influence translangagière? Plusieurs auteurs avancent l'idée selon laquelle les effets de l'influence translangagière sont plus évidents en compréhension qu'en production (Alonso, 2016; Ringbom, 2016). La tâche de jugement d'acceptabilité étant une tâche de compréhension, elle favorise donc la manifestation de l'influence translangagière.

Tournons-nous vers l'influence des variables linguistiques isolées au moyen de notre analyse sémantique, variables que nous allons discuter au regard de celles isolées dans les études antérieures comme étant des prédicteurs de l'acquisition des différents sens d'un mot polysémique.

\subsection{Analyse sémantique et la prototypicalité}

L'analyse sémantique que nous avons proposée est une analyse monosémique dans laquelle aucun des sens du verbe prendre n'est plus prototypique qu'un autre : le noyau de sens proposé relève d'un niveau de représentation cognitive, transcende l'ensemble des acceptions du verbe et ne correspond à aucune des acceptions en particulier. Cependant, les résultats aux tâches expérimentales ont tout de même montré que pour l'ensemble des apprenants, qu'ils soient anglophones ou allophones, les acceptions pour lesquelles le contact est fenêtré sont celles qui donnent lieu au plus grand nombre d'occurrences du verbe prendre à la tâche de jugement d'acceptabilité (71,7\%) et aussi, celles qui caractérisent $96,0 \%$ des acceptions prédicatives produites à la tâche de production. En acquisition des langues secondes, les résultats de plusieurs recherches ont révélé que le sens prototypique d'un mot polysémique est plus facilement acquis qu'un sens qui ne l'est pas (p.ex. Ijaz, 1986; Verspoor \& Lowie; 2003; Morimoto \& Loewen; 2007; Tyler, 2012). Pour définir la prototypicalité, les auteurs mettent de l'avant le caractère littéral, concret et fréquent du sens prototypique. Pour le verbe prendre, on pourrait donc postuler que, de par les premières entrées des dictionnaires ainsi que les analyses du verbe prendre proposées par d'autres auteurs (p.ex. Picoche, 1991; Peeters \& Eiszele, 1993), le sens prototypique serait une acception de type saisir comme dans Pierre prend un livre sur l'étagère. Selon notre analyse sémantique, ce sens correspond aux acceptions pour lesquelles le contact est fenêtré et se fait par saisie.

Or, les résultats que nous avons obtenus ne vont même pas dans le même sens. À la tâche de jugement d'acceptabilité, lorsque le contact est fenêtré et qu'il se fait par localisation, tous les participants ont 5,16 fois plus de chances $(\mathrm{p}<0,001)$ de choisir le verbe prendre (avec ou sans synonyme) par rapport au synonyme seul que pour toute autre acception du verbe. De plus, à la tâche de production écrite, où $96,0 \%$ des acceptions produites constituent des cas pour lesquels le contact est fenêtré, il y a autant de cas pour lesquels le contact se fait par localisation que ceux pour lesquels il se fait par saisie.

Comment alors expliquer qu'à la tâche de jugement d'acceptabilité les apprenants choisissent le verbe prendre (avec ou sans synonyme) dans $64,7 \%$ des cas lorsque le contact est fenêtré et se fait par saisie, tandis que dans les cas pour lesquels le contact est fenêtré et se fait par localisation, ils le choisissent dans $84,6 \%$ des cas? 
Étudions les trois exemples suivants. L'exemple en (28) est un exemple pour lequel le contact se fait par saisie et ceux en (29) et (30) des exemples pour lequel le contact se fait par localisation. Les phrases en (a) sont les phrases avec prendre et celles en (b), les phrases avec un synonyme.

a- Marie prend toujours la main de son fils avant de traverser la rue.

b- Marie tient toujours la main de son fils avant de traverser la rue.

a- Depuis ce temps, nous ne prenons plus le train pour aller au travail.

b- Depuis ce temps, nous n'empruntons plus le train pour aller au travail.

a- L'ennemi a pris la forteresse il y a trois jours.

b- L'ennemi a envahi la forteresse il y a trois jours.

Nous faisons donc l'hypothèse que les participants considèrent que tenir constitue un meilleur synonyme pour prendre dans le contexte en (28-b), que emprunter ou envahir, dans les exemples en (29-b) et (30-b). ${ }^{10}$ Toutefois, ces synonymes ne peuvent pas être mis sur le même plan, car on note une différence marquée en ce qui a trait à leur fréquence respective. Cette différence dans le choix du verbe prendre semble donc être liée non à la façon dont le contact se réalise, mais à la fréquence du synonyme proposé. Par conséquent, ce résultat pourrait être simplement lié aux liens de synonymie que nous avons choisis à la tâche de jugement, et non à la polysémie du verbe prendre à proprement parler.

Revenons aux résultats de la tâche de production écrite, tâche dans laquelle les liens de synonymie n'influencent pas la performance des apprenants. Si nous examinons la distribution pour les deux types de contact, lorsque le contact est fenêtré, nous observons que dans 57,2\% des acceptions celui-ci se fait par saisie et que dans $42,8 \%$ des acceptions il se fait par localisation. Cette différence n'est pas significative, comme le fait ressortir l'analyse présentée dans la partie 3.2.1.

Considérés ensemble, ces résultats nous montrent que ce n'est ni la saisie (comme nous le laissent croire les données dictionnairiques et les études antérieures), ni la localisation (comme semblaient le montrer les résultats à la tâche de jugement d'acceptabilité) qui constitue le sens premier du verbe prendre. Pour les participants à notre étude, ce qui est prototypique avec le verbe prendre est donc la notion de contact, notion qui ne correspond à aucune des acceptions spécifiques du verbe (c'est-à-dire celles qui actualisent un contact par saisie ou par localisation), mais qui transcende l'ensemble des acceptions de prendre. Ce sont donc les acceptions pour lesquelles le contact est fenêtré qui favorisent l'emploi du verbe prendre chez nos participants aux deux tâches expérimentales et qui pourraient ainsi constituer une sorte d'emploi prototypique.

\subsection{Le caractère concret ou abstrait d'une acception}

Comme nous l'avons soulevé dans notre analyse sémantique de la polysémie du verbe prendre, nous avons effectué un typage sémantique afin d'étiqueter les différents éléments du co-texte du verbe prendre selon les traits sémantiques qui les caractérisent. Le premier niveau consistait à distinguer les entités physiques des entités abstraites. Or, cette variable, même si elle est assez importante dans les études portant sur l'acquisition du vocabulaire, n'a pas une grande importance dans le noyau de sens que nous avons proposé pour rendre compte de la polysémie du verbe prendre. Toutefois, nous avons réanalysé nos données en répartissant les acceptions prédicatives en deux catégories : les acceptions concrètes et les acceptions abstraites. Nous définissons une acception concrète comme étant une acception pour laquelle il y a un contact physique entre le sujet et le complément direct (pour les acceptions transitives) ou encore entre les différents composants du sujet (pour les acceptions intransitives).

Ainsi, nous avons effectué une analyse de régression logistique dans laquelle les variables indépendantes sont le niveau de compétence langagière en français L2, la langue maternelle des apprenants et le caractère concret ou abstrait d'une acception (tableau 5). 
Tableau 5 : Régression logistique avec la variable concret/abstrait

\begin{tabular}{llll}
\hline & Rapport de chances & Erreur type & Sig. \\
\hline Constante & 1,50 & 0,26 & 0,03 \\
Niveau & 0,97 & 0,01 & 0,001 \\
$\begin{array}{l}\text { Langue } \\
\text { (Allophone-réf.) }\end{array}$ & 0,82 & 0,09 & 0,07 \\
$\begin{array}{l}\text { Concret/abstrait } \\
\text { (abstrait-réf.) }\end{array}$ & 1,44 & 0,16 & 0,002 \\
$\begin{array}{l}\text { Langue \# } \\
\begin{array}{l}\text { Concret/abstrait } \\
\text { Anglophone \# concret }\end{array}\end{array}$ & 1,05 & 0,15 & 0,745 \\
\end{tabular}

Notes : nombre d'observations $=3505 ; \log$ vraisemblance $=-2403,88, \chi^{2}=50,08, p<0,001 ;$ pseudo $R^{2}=0,0103$; réf. = catégorie de référence pour les variables catégorielles

Les résultats de cette analyse nous montrent que tous les apprenants, quelle que soit leur langue maternelle, ont 1,44 fois plus de chances de choisir le verbe prendre (avec ou sans synonyme) lorsque l'acception est une acception concrète. Ce résultat rejoint donc ceux des études antérieures qui soutiennent l'idée qu'un sens concret est plus facilement acquis qu'un sens abstrait.

Cependant, si l'on compare le modèle présenté dans le tableau 4 (variables issues de l'analyse sémantique) et celui dans le tableau 5 (avec la variable concret/abstrait) afin d'évaluer lequel s'ajuste le mieux aux données et a ainsi le plus de pouvoir explicatif, on peut avoir une idée plus fine de la portée des deux analyses. Pour ce faire, nous avons donc comparé les deux modèles en utilisant les scores de log vraisemblance de chacun des modèles. ${ }^{11}$ Selon Bressoux (2008), la comparaison des modèles de régression logistique se fait à l'aide du calcul suivant : -2 (log vraisemblance [modèle 1]) - -2(log vraisemblance [modèle 2]). Puis, on soustrait les degrés de liberté du modèle 1(5) des degrés de liberté du modèle $2(13)$. Le résultat obtenu est une valeur qui suit une distribution de khi carré, dans notre cas : $\chi^{2}$ $(7)=444,31, p<0,001$, valeur qui est significative. Nous pouvons donc conclure que le modèle avec les variables issues de notre analyse sémantique est significativement meilleur que celui avec la variable liée au caractère concret ou abstrait de l'acception.

En somme, nous ne pouvons pas infirmer cette idée selon laquelle une acception concrète sera acquise plus facilement qu'une acception abstraite. Nous pouvons cependant y mettre un bémol. La différence entre les deux modèles montre que l'analyse sémantique que nous avons proposée rend mieux compte de la variance dans le choix du verbe prendre à la tâche de jugement d'acceptabilité que le caractère concret ou abstrait d'une acception. Plutôt que le caractère concret ou abstrait d'une acception, c'est donc la saillance de l'élément fenêtré dans les différents sens du verbe prendre qui permet de mieux expliquer que certaines acceptions sont plus facilement acquises.

\section{Conclusion : applications à la didactique des langues secondes}

Consacré à la polysémie du verbe prendre et à l'acquisition des différents sens de ce verbe par des apprenants du français L2, ce travail permet de mettre au jour les variables individuelles et linguistiques qui peuvent prédire l'acquisition des différentes acceptions de mots hautement polysémiques en L2.

D'abord, pour la population anglophone, l'influence translangagière est bien présente à la tâche de jugement d'acceptabilité, mais pas à celle de production écrite. Ce résultat rejoint celui des études qui montrent qui l'influence translangagière se manifeste davantage en compréhension qu'en production. Il s'agira de travailler explicitement sur les points d'interférence afin de développer des représentations sémantiques indépendantes de la L1. Il y aurait également lieu de favoriser des connaissances métalinguistiques relativement aux points d'interférence, afin d'amener les apprenants à contrer les effets de l'influence translangagière, surtout en compréhension.

Quant aux variables linguistiques, revenons sur celles liées à la prototypicalité et au caractère concret ou abstrait de l'acception. Plusieurs études, dans lesquelles est présente une intervention pédagogique ayant pour but de favoriser l'apprentissage des différents sens d'un mot polysémique, ont été menées dans l'optique d'une analyse prototypique de la polysémie ancrée en linguistique cognitive (p. ex. Verspoor \& Lowie, 2003; Csábi, 2004). De manière générale, dans ces études, il s'agit d'amener les apprenants à maîtriser différents sens d'un mot polysémique en partant d'un sens prototypique. Ainsi, la prototypicalité est considérée comme étant un phénomène lexical : le sens prototypique, souvent lié à un élément lexical concret, sera le plus facile à apprendre. 
Or, le pouvoir prédictif de notre analyse monosémique, dans laquelle aucun des sens du verbe n'est plus prototypique que l'autre, nous amène à proposer de revoir la notion de prototypicalité en ce qui a trait à la façon dont on l'applique à la didactique des langues secondes. Ce qui est prototypique est conceptuel et non lexical, comme le concept du contact pour le verbe prendre. Plusieurs études semblent aller dans ce sens. Morimoto et Loewen (2007), par exemple, ont montré que des schémas conceptuels graphiques (comparables à un noyau de sens) favorisent l'apprentissage des différents sens de deux mots polysémiques (break et over) chez des apprenants japonais de l'anglais L2. De plus, l'étude de Degani, Tseng et Tokowicz (2014) a montré que le fait d'apprendre les traductions de mots ambigus en même temps facilite leur acquisition (p. 759). En effet, selon les auteurs, le fait de présenter les différents sens d'un mot polysémique au même moment aide les apprenants à distinguer les sens les uns des autres (p. 759). Qui plus est, apprendre en même temps les multiples sens des mots ambigus évite à l'apprenant de reconstruire sa représentation sémantique d'un mot comme c'est le cas pour les mots dont il apprend les différents sens de façon successive (p. 761). Nous proposons alors qu'un concept clé ou prototypique, tel que le contact avec le verbe prendre, pourrait aider les apprenants à comprendre plus facilement les liens entre les différents sens d'un verbe hautement polysémique.

Puis, certaines études ont proposé qu'une acception concrète soit plus facilement maîtrisée qu'une acception abstraite. Toutefois, nous avons montré que, pour la polysémie du verbe prendre, une variable liée au caractère concret ou abstrait d'une acception avait beaucoup moins de pouvoir explicatif que l'analyse sémantique que nous avons proposée. Par ailleurs, nous avons vu que le contact constitue un concept prototypique pour le verbe prendre, concept qui ne correspond à aucune acception en particulier, mais qui est présent dans l'ensemble de ces acceptions. Dans les acceptions pour lesquelles le contact est fenêtré, sont incluses des acceptions qui sont concrètes ou abstraites, montrant également que le caractère concret ou abstrait d'une acception a relativement peu d'importance dans la détermination des sens qui sera acquis en premier.

Enfin, cette étude nous a permis de mettre en évidence l'apport d'une analyse monosémique par rapport à la compréhension des phénomènes liés à l'acquisition des différents sens d'un mot polysémique en L2.

\section{Références bibliographiques}

Altenberg, B., \& Granger, S. (2001). The grammatical and lexical patterning of MAKE in native and non-native student writing. Applied Linguistics, 22, 173-195.

Alonso, R. A. (Dir.) (2016). Crosslinguistic influence in second language acquisition. Bristol: Multilingual Matters.

Berthaud, S., \& Antonijevic, S. (2012). L2 acquisition of verbal constructions: Expressing motion in L2 French and English. Dans M. Bouveret \& D. Legallois (Dir.), Constructions in French (pp. 155-174). Amsterdam/ Philadelphia: John Benjamins Publishing Company.

Bolly, C. (2007). La notion de compétence "phraséologique": Verbes à haute fréquence et corpus d'apprenants anglophones avancés. Dans I. González Rey (Dir.), Les expressions figées en didactique des langues étrangères (pp. 35-51). Cortil-Wodon: E.M.E. \& InterCommunications.

Bolly, C. (2009). The acquisition of phraseological units by advanced learners of French as an L2: High-frequency verbs and learner corpora. Dans E. Labeau \& F. Myles (Dir.), The advanced learner variety: The case of French (pp. 199-220). Bern, Berlin: Peter Lang.

Bressoux, P. (2008). Modélisation statistique appliquée aux sciences sociales. Bruxelles: De Boeck.

Crossley, S., Salsbury, T., \& McNamara, D. (2010). The development of polysemy and frequency use in English second language speakers. Language Learning, 60, 573-605.

Csábi, S. (2004). A cognitive linguistic view of polysemy in English and its implications for teaching. Dans M. Achard \& S. Niemier (Dir.), Cognitive Linguistics, second language acquisition, and foreign language teaching. New York: Mouton de Gruyter.

Degani, T., \& Tokowicz, N. (2010). Ambiguous words are harder to learn. Bilingualism: Language and Cognition, 13, 299-314.

Degani, T., Tseng, A. M., \& Tokowicz, N. (2014). Together or apart: Learning of translation-ambiguous words. Bilingualism: Language and Cognition, 17, 749-765.

El Guedri, J. (2011). Le verbe 'prendre': Analyse syntactico-sémantique en vue de l'élaboration d'un dictionnaire électronique. (Thèse de doctorat),Université Paris XIII.

Elston-Güttler, K., \& Williams, J. N. (2008). L1 polysemy affects L2 meaning interpretation: Evidence for L1 concepts active during L2 reading. Second Language Research, 24, 167-187.

Gullberg, M. (2009a). Gestures and the development of semantic representations in first and second language acquisition. Acquisition et interaction en langue étrangère, 1, 117-139.

Gullberg, M. (2009b). Reconstructing verb meaning in a second language: How English speakers of L2 Dutch talk and gesture about placement. Annual Review of Applied Linguistics, 7, 221-224.

Hayashi, M. (2008). The acquisition of the verb "make" by Japanese learners of English. The Annals of Gifu Shotoku Gakuen University, 55, 25-47.

Hayashi, M., \& Marks, T. (2012). On perception of the verb "give" by Japanese learners of English. Ritsumeikan Studies in Language and Culture, 23, 159-174.

Ijaz, H. (1986). Linguistic and cognitive determinants of lexical acquisition in a second language. Language Learning, 36, $402-451$.

Jarvis, S. (2000). Methodological rigor in the study of transfer: Identifying L1 influence in the interlanguage lexicon. Language Learning, 50, 245-309.

Jarvis, S., \& Pavlenko, A. (2008). Crosslinguistic influence in language and cognition. New York: Routledge. 
Jiang, N. (2002). Form-meaning mapping in vocabulary acquisition in a second language. Studies in Second Language Acquisition, 24, 617-637.

Jiang, N. (2004). Semantic transfer and its implications for vocabulary teaching in a second language. The Modern Language Journal, 88, 416-432.

Kleiber, G. (1999). Problèmes de sémantique: la polysémie en questions. Villeneuve d'Ascq: Presses universitaires du Septentrion.

Kleiber, G. (2005). Quand y a t-il sens multiple? Le critère referentiel en question. Dans O. Soutet (Dir.), La Polysémie (pp. 51-74). Paris: Presses de l'Université Paris-Sorbonne.

Laufer, B. (1990). Why are some words more difficult than others? Some intralexical factors that affect the learning of words. International Review of Applied Linguistics, 28, 293-307.

Lennon, P. (1996). Getting 'easy' verbs wrong at the advanced level. IRAL, 34, 23-36.

Manes Gallo, M. C., \& Rouault, J. (2010). Variations syntactico-sémantiques sur la polysémie du verbe 'prendre'. Liens linguistiques, 90, 427-443.

Miller, G. A. (1995). WordNet: A lexical database for English. Communications of the ACM, 38, 39-41.

Morimoto, S., \& Loewen, S. (2007). A comparaison of the effects of image-schema-based instruction and translation-based instruction on the acquisition of L2 polysemous words. Language Teaching Research, 11, 347-372.

Paillard, D. (2001). À propos des verbes polysémiques: identité sémantique et principes de variation. Dans J. François (Dir.), Syntaxe \& Sémantique, Sémantique du lexique verbal (pp. 99-120). Caen: Centre de recherche inter-langues sur la signification en contexte.

Peeters, B., \& Eiszele, A. (1993). Le verbe 'prendre' pris au sérieux. Cahiers de lexicologie, 62, 169-184.

Picoche, J. (1991). Structure sémantique du verbe prendre en français et en moyen français. Linx, 3, 161-177.

Ravin, Y., \& Leacock, C. (2000). Polysemy: theoretical and computational approaches. Oxford; Toronto: Oxford University Press.

Redmond, L. (2017). Acquisition de la polysémie du verbe prendre par des apprenants du français langue seconde (Thèse de doctorat en co-tutelle), Université du Québec à Montréal et Université Bordeaux Montaigne.

Ringbom, H. (2016). Comprehension, learning and production of foreign languages: The role of transfer. Dans R. A. Alonso (Dir.), Crosslinguistic influence in second language acquisition (pp. 38-52). Bristol: Multilingual Matters.

Ruhl, C. (1989). On monosemy: A study in linguistic semantics. Stony Brook: State University of New York Press.

Saji, N., \& Imai, M. (2013). Evolution of verb meanings in children and L2 adult learners through reorganization of an entire semantic domain: The case of Chinese carry/hold verbs. Scientific Studies of Reading, 17, 71-88.

Senéchal, M. (2012). La polysémie des verbes à constructions locatives en français contemporain: Le cas des verbes à construction directe locative. (Thèse de doctorat), Université de Caen Basse-Normandie.

Talmy, L. (2000). Toward a cognitive semantics. Cambridge, MA: MIT Press.

Tyler, A. (2012). Spatial language, polysemy, and cross-linguistic semantic mismatches: Cognitive linguistics insights into challenges for second language learners. Spatial Cognition \& Computation, 12, 305-335.

Uçkun, B. (2012). Awareness of verb subcategorization probabilities with polysemous verbs: The second language situation. System, 40, 360-375.

Verspoor, M., \& Lowie, W. (2003). Making sense of polysemous words. Language Learning, 53, 547-586.

Viberg, A. K. (2002). Basic verbs in second language acquisition. Revue française de linguistique appliquée, 7, 51-69.

Victorri, B., \& Fuchs, C. (1996). La polysémie: construction dynamique du sens. Paris: Hermès.

\footnotetext{
${ }^{1}$ Nous remercions les relecteurs anonymes pour leurs commentaires sur une version antérieure de cet article.

${ }^{2}$ Ce travail s'inscrit dans le cadre d'un projet plus large, projet dans lequel nous avons analysé les différents emplois du verbe prendre, à savoir les constructions à verbe support, les locutions verbales et les acceptions prédicatives (voir Redmond (2017)).

${ }^{3}$ Nous avons consulté le Grand dictionnaire de la langue française (Larousse) le Trésor de la langue française informatisé et le Grand Robert électronique.

${ }^{4}$ Nous nous sommes fondées, entre autres, sur la base de données lexicales WordNet (Miller, 1995).

${ }^{5}$ Nous avons consulté le Dictionnaire Électronique des Synonymes ainsi que les synonymes présentés dans les autres dictionnaires consultés.

${ }^{6}$ Les L1 des apprenants allophones varient; nous en avons dénombré vingt.

${ }^{7}$ Le test comportait un total de 34 items. Pour les fins de cet article, nous avons exclu les constructions à verbe support et les emplois erronés du verbe prendre calqués sur le verbe take (items qui permettent d'approfondir la notion d'influence translangagière) pour nous concentrer sur les 24 acceptions prédicatives du verbe prendre.

${ }^{8}$ Le score de log vraisemblance et les degrés de liberté sont obtenus au moyen de la commande de post-estimation (estat ic) dans le logiciel Stata.

${ }^{9}$ Les 73 allophones ont vingt langues maternelles différentes et constituent ainsi un ensemble hétérogène et donc, d'une certaine façon, un groupe de contrôle.

${ }^{10}$ Selon lexique.org, la fréquence de chacun de ces verbes (calculée sur un million d'occurrences) est la suivante : tenir $(104,52)$, emprunter $(6,74)$, envahir $(5,5)$.

${ }^{11} \mathrm{Au}$ total, les apprenants ont produit 895 phrases. Nous nous concentrons sur les 660 acceptions prédicatives. Nous avons exclu les locutions verbales, les constructions à verbe support et les phrases comportant une erreur sémantique avec le verbe prendre.
} 